\title{
An advanced endoscopic surgery robotic platform for removal of early-stage gastrointestinal cancer using endoscopic submucosal dissection
}

\author{
Ka Chun Lau ${ }^{1,2, *}$, Yeung Yam ${ }^{1,2,3}$ and Philip Wai Yan Chiu ${ }^{2,3,4}$ \\ ${ }^{1}$ Department of Mechanical and Automation Engineering, The Chinese University of Hong Kong, Hong Kong, People's \\ Republic of China \\ ${ }^{2}$ Multi-scale Medical Robotics Center, The Chinese University of Hong Kong, Hong Kong, People's Republic of China \\ ${ }^{3}$ Chow Yuk Ho Technology Centre for Innovative Medicine, Faculty of Medicine, The Chinese University of Hong Kong, \\ Hong Kong, People's Republic of China \\ ${ }^{4}$ Department of Surgery, Faculty of Medicine, The Chinese University of Hong Kong, Hong Kong, People's Republic of China
}

\begin{abstract}
Two gastrointestinal (GI) cancers, stomach and colorectal cancer, have the fifth and third highest incident rates and the fourth and second highest mortality rates among all cancers, respectively. Combined, they had 2.8 million new cases and 1.6 million deaths annually. Fortunately, early-stage GI cancer has a high five-year survival rate if the tumour can be removed completely. Endoscopic Submucosal Dissection (ESD) is the gold standard for the removal of early-stage GI cancer as it has a high rate of en-bloc resection and a low rate of recurrence. However, ESD is a very technically challenging surgical operation. It has a relatively high rate of failure, including perforation of the stomach or colon. Therefore, a surgical robotic platform with high dexterity and better ergonomics is in high demand, which can ease the technical problems induced by conventional ESD procedures. In our previous study, a prototype of an endoscopic surgery robotic platform was built and the preliminary experimental results showed that the dual flexible arm robotic platform with wire-driven continuum structure was capable of increasing the efficiency and safety in performing ESD under the master-slave controlling scheme. An advanced robotic platform was built based on this prototype to achieve higher flexibility and production requirements. The design of these two platforms and experimental results will be presented in detail.
\end{abstract}

KEYWORDS Surgical robot; endoscopic surgery; flexible robot; continuum structure; tendon-sheath mechanism; Endoscopic Submucosal Dissection; early-stage GI cancer

CONTACT Ka Chun Lau kclau@mrc-cuhk.com

Received 7 March 2021

\section{Introduction}

\section{1. Background information on gastrointestinal cancers}

Gastrointestinal (GI) cancers, including stomach and colorectal cancer, are the most common cancers worldwide nowadays. In 2020, stomach cancer had the fifth and the fourth highest incident and mortality rate among all cancers, respectively; colorectal cancer had the fourth and second highest incident and mortality rate respectively (International Agency for Research on Cancer, 2020). Stomach and colorectal cancers have, combined, 2.8 million new cases and 1.6 million deaths annually. Especially in China, GI cancer is the deadliest type of cancer. It caused 0.66 million deaths in 2020 (International Agency for Research on Cancer, 2020). Fortunately, the five-year survival rate of early-stage GI cancer is relatively higher than that of other cancers, which can be higher than 90\% (Cenitagoya, Bergh, and Klinger-Roitman, 1998). Hence, early screening of the GI tract is an important social practice.

Currently, an endoscopic surgical procedure called Endoscopic Submucosal Dissection (ESD) has been widely used for the removal of early-stage GI cancer. This is because, firstly, it can be used to treat larger tumours, such as those larger than $20 \mathrm{~mm}$ in diameter (Yoshida et al., 2013). Secondly, ESD has a very high rate of en-bloc (complete) resection, about 80-98.9\% (Isomoto et al., 2009; Zhou et al., 2009; Yoshida et al., 2010), and a very low rate of recurrence, comparable to a traditional procedure called Endoscopic Mucosal Resection (EMR) (Oka et al., 2006). Since most early-stage GI cancers grow within the mucosa tissue layer of the GI layer, i.e., the top layer, ESD aims to remove the tissue layer deeper than that, which is the submucosal tissue layer.

ESD is a minimally invasive surgery (MIS). In the ESD procedure, only two surgical tools will be used: an endoscope and an electrocautery knife. Before the start of the surgical procedure, an endoscope will be inserted into the target organ, for instance, the stomach or colon, through a natural opening (here the mouth or anus, respectively) to inspect the actual surgical location. After that, an electrocautery knife will be inserted through the channel of the endoscope. ESD is then started and the whole procedure can be divided into six steps (Asano, 2012).

Firstly, an electrocautery knife, for example, the Dual Knife from Olympus, is used to mark dots around the tumour with about a $3 \mathrm{~mm}$ margin. This procedure helps the surgeon to identify the location of the tumour clearly during the surgery. Secondly, surgeons inject indigo-dyed saline solution between the submucosal tissue layer and the muscle layer. The dyed saline helps to better highlight the 
dissection area, and achieve a sustained mucosal elevation for dissection. Thirdly, a partial incision is made along with the markings in preparation for the dissection. After that, the submucosal tissue layer is dissected using the electrocautery knife inside of the incision and along the incision margin. This process is repeated until the whole specimen is dissected completely (Gotoda et al., 2006).

Compared to open surgery, MIS is less invasive since the procedure is not performed through a large open incision. It is also less expensive and can better preserve the biological function of organs. However, performing MIS in the GI tract, especially ESD, is a very technically challenging procedure since it is associated with a higher risk of complications and requires many years of training (Teoh et al., 2010). There are three shortcomings that can be seen in ESD. First of all, only one accessory (the electrocautery knife) is used during the whole procedure. There is a lack of retraction of tissue during dissection. Without retraction of tissue, it is difficult to maintain the accuracy and efficiency during dissection. Secondly, the motion of the electrocautery knife, the left-and-right and up-and-down motion, is almost totally driven by the endoscope. Only the translational, forward-and-backward motion can be controlled independently. This induces a coupling motion between the electrocautery knife and the camera of the endoscope, i.e., the view point of the camera moves together with the electrocautery knife. Most vision will be blocked by the endoscope itself during dissection. Thirdly, it is difficult to maintain the tip of the endoscope in a stable position inside the hollow viscus, which affects precision control of the endoscope (Morgan, 2017). The ESD procedure can be summarised in one sentence: a surgeon mounted a knife on his head to dissect the tumour by turning his neck.

Therefore, ESD is a very time-consuming surgical procedure. The average operation time is about $58.5 \pm 28.7$ minutes for tumours smaller than $10 \mathrm{~mm}$ in diameter; and about $123.8 \pm 101.4$ minutes for tumours larger than 21 $\mathrm{mm}$ in diameter (Oka et al., 2006). The lengthy operation time gives a challenge to surgeons. Moreover, the bleeding and perforation rate of ESD is high. The incidence of perforation in some studies was between 10-53.8\% (Oka et al., 2006; Asano, 2012). Perforation is a serious problem that must be handled carefully, since carbon dioxide is used as the insufflation gas to enlarge the organ. If the organ is perforated, the carbon dioxide will be absorbed rapidly by the blood vessels and thus cause exacerbation (Gotoda et al., 2006). If the bleeding or perforation is too serious, emergency open surgery will be needed to close the wound.

\subsection{Motivation}

From a clinical perspective, finding a better treatment method is always our target. In order to further reduce the pain and recovery time of patients, many open surgeries already moved to MIS in recent years. However, MIS still has many complications, such as a long training time. Nowadays, our medical system is overloaded. We do not have enough doctors, nurses, and even hospital space. There is great demand for a faster medical treatment method to reduce pressure on the medical system, while maintaining a high level of safety at the same time. Robotising endoscopic surgery would be one possible solution to this problem. Robots could manipulate different instruments at the same time, which could speed up the process. Robots could be very stable, compared to humans. The accuracy of a robotic arm is higher than a human arm as well.

From an engineering perspective, robotising endoscopic surgery is an emerging research field. Laparoscopic surgery has been robotised for many years. The most famous example is the da Vinci Surgical System from Intuitive Surgical. Since it was released in 1998, over a million cases have been completed by this system. However, the development of robotised endoscopic surgery is lagging far behind.

Unlike laparoscopic surgery, the instruments used in endoscopic surgery have to be flexible and soft, as they need to reach the inner organ through the long twists and turns of a channel (e.g., esophageal and intestine). Controlling flexible and soft robotic arms is challenging as it could be modelled as an infinite number of degree-of-freedom (DoF) bodies. We usually add some constraints to the structure so that it behaves more reasonably. Meanwhile, it is very challenging to design a flexible robotic arm that is soft enough to reach the target, but also strong enough to lift up tissue for dissection.

Moreover, the channel inside the human body is narrow. Therefore, the working area is small, compared to laparoscopic surgery, and there is only one approach angle that can be achieved. How to maintain enough DoF while keeping the size of the robotic arm small is always an interesting and hot topic in academic research.

Last but not least, ESD is a very effective surgical procedure for the treatment of early-stage GI cancer. If the technical problems of performing ESD can be solved, more surgery can be performed, and thus more patients will be benefited.

\subsection{Related works}

Dual robotic arm configuration is a common design for an endoscopic surgery robotic platform. In general, these kinds of robotic platforms can be divided into two groups: purely mechanical systems and robotised systems.

For a purely mechanical system, no motorised or electrical components are used. Cobra, developed by USGI Medical, is designed from its endoscopic platform TransPort. Cobra has two robotic arms and a camera channel which also can be bent in two directions. All these DoFs are controlled by a handheld controller directly connected by wires (Swanstrom et al., 2005). Olympus also developed a similar direct-driven robotic platform, 
called EndoSamurai (Spaun et al., 2009). EndoSamurai has five DoFs on each robotic arm. At least two operators are needed to control all the DoFs of this system and experimental results showed its effectiveness in both ex-vivo and in-vivo experiments over the conventional endoscope. However, these pure mechanical systems have two major drawbacks. Firstly, not all DoFs can be controlled simultaneously, even with two operators. The usefulness of using a robotic arm to perform endoscopic surgery cannot be manifested. Secondly, since the robotic arms of these systems cannot be retracted before inserting into human body, there is increased difficulty to insertion and increased risk of damaging the inner organs. For a more practical and useful solution, a robotised system is superior to a purely mechanical system.

For a robotised system, all the DoFs of the robotic arms are controlled by motors. The STRAS, from the University of Strasbourg France, is a multi-functional endoscopic robotic platform (De Donno et al., 2013). It can be used in normal endoscopic surgery, Natural Orifice Transluminal Endoscopic Surgery (NOTES), and Single Port Surgery (SPS). It has a total of 10 DoFs. It has a distal articulated cap that can be opened into the left and right half which can provide smooth insertion and increases the separation distance of the two robotic arms for better triangulation manipulation. The Master and Slave Transluminal Endoscopic Robot (MASTER) developed by Nanyang Technological University (Cao et al., 2019) and the K-Flex system developed by Korea Advanced Institute of Science and Technology (Ahn et al., 2021) also focus on endoscopic surgery. They depend on a specially designed endoscope as a carrier to build the robotic arms to the destined location. Their overall structure complexity is high, while their size is not typically very small.

CYCLOPS, developed by Imperial College London, is an endoscopic robotic platform in which the controlling wires are not anchored at the distal end. The controlling wires of this robotic platform are anchored inside a very large bowel that is placed around the robotic arms (Mylonas et al., 2014). This novel mechanism enables the robotic arms to provide much greater lifting force than the conventional endoscopic robotic arm. However, the size of the large bowel becomes a constraint when this robotic platform is operating inside the human body.

\section{Design of the robotic platform}

\subsection{Design requirements}

As discussed in the previous section, there are three technical problems in using conventional endoscopic tools, namely an endoscope and electrocautery knife, to perform ESD. According to these problems, there are three needs we can define when designing the robotic platform.

Firstly, the motion between the camera and the tools must be decoupled. As mentioned, conventional
ESD procedures usually suffer blocking of vision during dissection. Surgeons are unable to monitor the dissection area during dissection and thus a high perforation rate is the outcome. If their movement can be decoupled, a more accurate dissection can be achieved. Secondly, the tissue layer must be retracted. With only one tool (electrocautery knife) in operation during the whole procedure, there is no tension on the soft tissue layer; like trying to cut a steak without stabilising it with a fork, this affects the accuracy and efficiency of the operation of the knife. Hence, if the tissue layer can be retracted, surgeons can provide a safer and faster dissection. Thirdly, the robotic platform should have a cognitive control interface. Controlling an endoscope needs many years of training as the motion of the endoscope is not relative to the camera view. When the endoscope is twisted or turned by over 180 degrees, the endoscope's direction of motion will be reversed. Having an easy-to-learn controlling interface can greatly reduce the learning curve for surgeons.

In light of these requirements, the must-have features of the robotic platform are as listed here: (1) a robotic arm with dissection ability, (2) a robotic arm with retraction and lifting ability, (3) the ability to operate these two robotic arms independently, (4) these two robotic arms must be flexible, (5) the DoF of the master console must match the motion of the robotic arms.

\subsection{Robotic platform overview}

In this section, the design of the prototype of the robotic platform and the advanced robotic platform will be presented. As shown in the below figure, the robotic system can be divided into four components: master console, computer, driving unit, and a robotic arm.

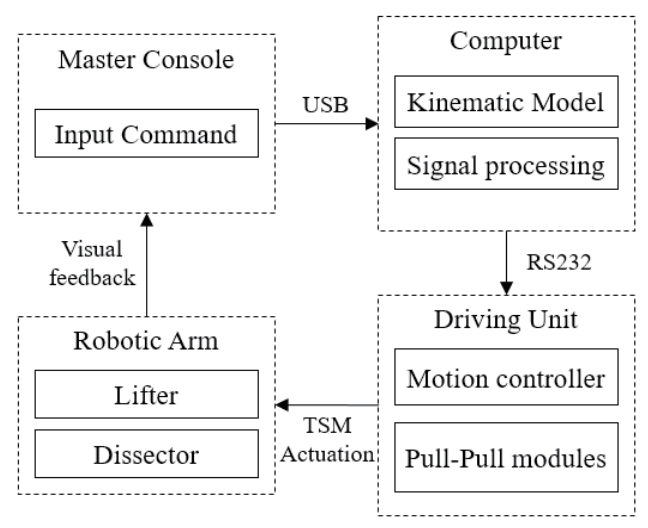

Figure 1. The components of the robotic system.

The overall controlling flow of this robotic platform is thus summarised. The master console receives the input command from the operator - in this case, the surgeon. The signal is then sent to a computer for processing. The desired motors' position and velocity, after calculation by the designated kinematic algorithm, are set by the computer. 
The tendon-sheath mechanism (TSM) is used as the power transmission, connecting the motors and the robotic arm so that power and action can be transmitted through the twists and turns of the channel. An endoscopic platform by USGI Medical, TransPort (green in Figure 2), is used as a carrier to bring the robotic arm into the human body. The two robotic arms, the lifter and dissector, are placed at the east and west side channels of the TransPort respectively. A camera is placed at the north channel, as shown in Figure 3. The operator can thus control the robotic arms through the view from the camera.

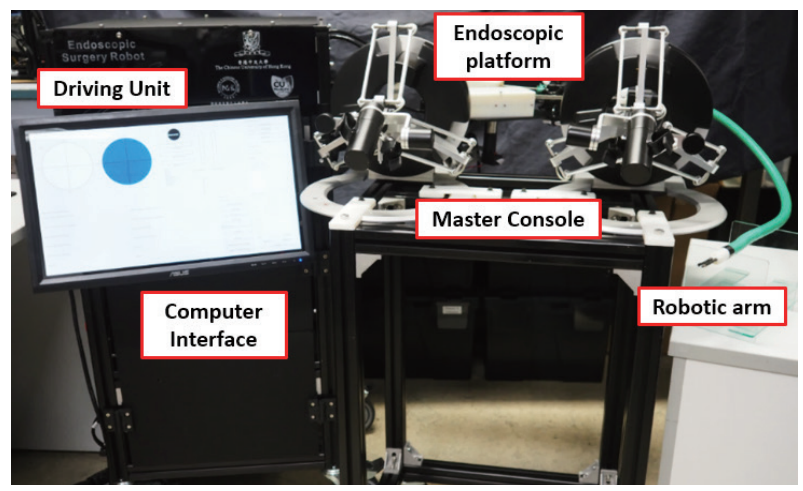

Figure 2. Overview of the robotic platform prototype.

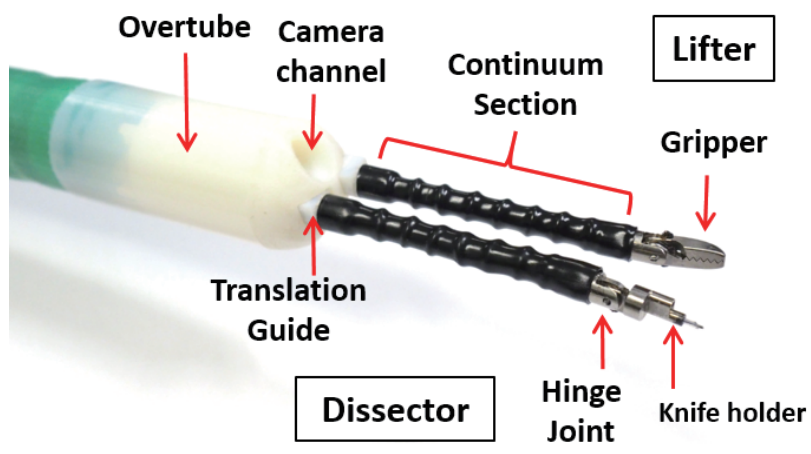

Figure 3. Overview of the robotic arm.

Basically, the lifter and the dissector have the same structure but with different end-manipulators. The continuum section is constructed with a lot of disks such that it can be bent in two directions: left-and-right, up-anddown. The continuum section is controlled through two pairs of wires. The bending direction depends on which pair of wires changes length. As illustrated in Figure 4, when the length of $\mathrm{A}$ is shorter than $\mathrm{B}$, then the continuum section will be bent towards the positive $\mathrm{x}$-axis and vice versa.

The lifter consists of a gripper that can grip and lift up the tissue layer to create retraction and tension. The dissector consists of a knife holder and a hinge joint to control the electrocautery knife for the dissection. There is a total of nine DoFs provided from the lifter and dissector, as shown in Figure 5. The lifter and dissector can be translated independently (DoFs 1 and 2). DoFs 3-6 is the bending motion of the continuum section of these two robotic arms respectively. The upper and lower jaw of the gripper of the lifter can be controlled independently (DoFs 8, 9) and the knife holder can provide one more DoF (DoFs 7).

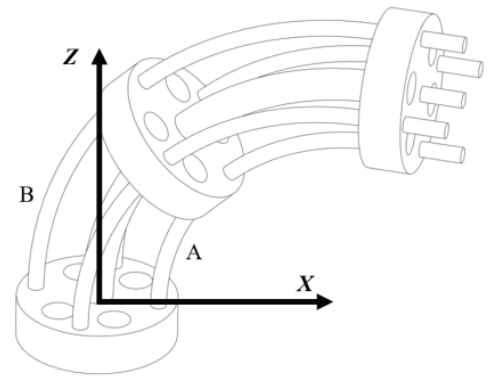

Figure 4. The structure of the continuum section.

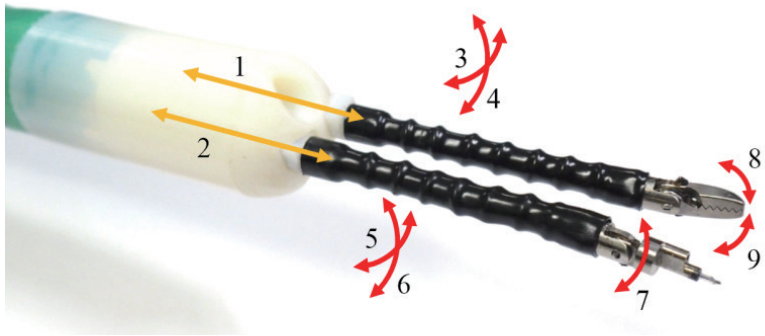

Figure 5. Illustration of the DoFs of the robotic arm.

The master console consists of two controllers, the left-handed and right-handed configuration Omega 7, provided by Force Dimension. Each controller has seven DoFs and four of them have force feedback (X-, Y-, Z-translation and gripper). The mapping between the controller and the robotic arms is shown in Figure 6. The X-, Y- and Z-translation of the controller are mapped to the tip position of the robotic arms and the pitch input is mapped to the pointing direction of the distal tip. The opening angle of the gripper is controlled through the gripper input of the controller directly.

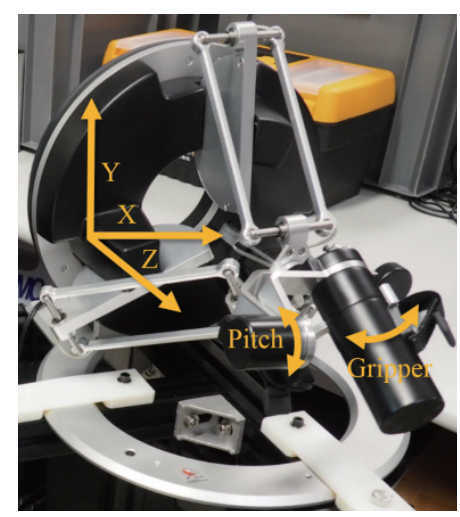

Figure 6. The control mapping between the controller and the robotic arm. 
This prototype had proved in our previous ex-vivo and animal studies that it could greatly reduce the time spent performing ESD and the risk of perforation (Lau et al., 2016, 2017). In order to enhance the commercialisation possibility of this platform, a more advanced robotic platform was designed, as shown in Figure 7.

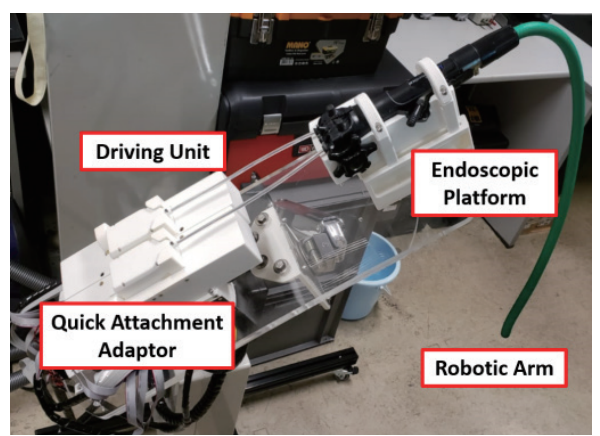

Figure 7 . The advanced endoscopic surgery robotic platform.

The robotic arms are still using the endoscopic platform as the carrier, while the control wire is not connected to the motor couplers directly. They are embedded into the quick attachment adaptor, such that the robotic arms can be installed or removed from the platform without any tools. There are four knots on the adaptor with which to control the four-DoFs of the robotic arms and those knots can be matched on the driving unit, as shown in Figure 8. There are two independent units on the driving unit to provide the translation motion of each of the robotic arms.

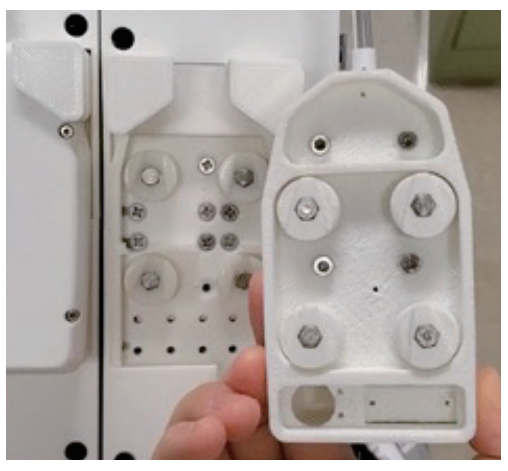

Figure 8 . The quick attachment adaptor of the robotic arm.

\subsection{Design and modelling of continuum structure}

The design of the continuum structure of the advanced robotic platform is enhanced such that it withstands the higher torque exerted on it. The bending mechanism has been changed from disks to a rolling gear joint. In short, the rolling gear joint can provide smooth bending along its bending direction, while also providing an anti-twisting function that increases the lifting force and stability. As shown in Figure 9, the rolling gear joint does not affect its flexibility, and the maximum bending angle is unchanged (120 degree), while the strength and anti-torque ability are increased.

The kinematic mapping of the continuum section has three domains, as illustrated in Figure 10. The first domain is the actuator space, i.e., the length of the wire. With different wire lengths, we can define the second domain, the configuration space, in which curvature and bending direction will be used to describe the shape of the continuum section. This mapping is called specific mapping, because this mapping is different for each design of the continuum section. For example, the continuum section used in this robotic platform has two pairs of wires. Therefore, there are four variables, which are wire lengths, in the actuator space. If the continuum section is driven by three wires, the actuator space will have three variables of wire length.

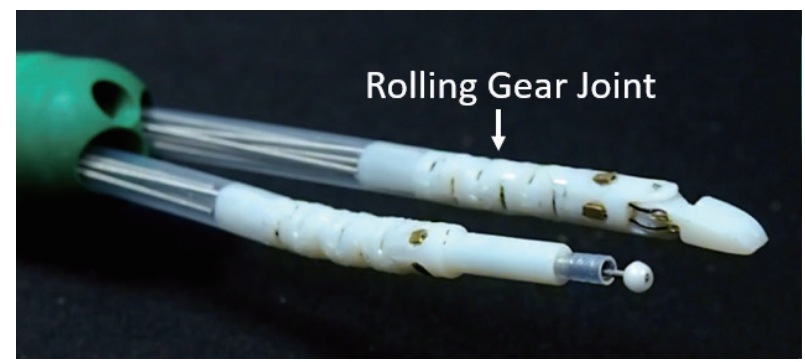

Figure 9. The rolling gear joint of the advanced robotic platform.

After that, the mapping from the configuration space to task space is called independent mapping as this mapping is the same for all continuum sections. The tip position and orientation can be calculated from the shape of

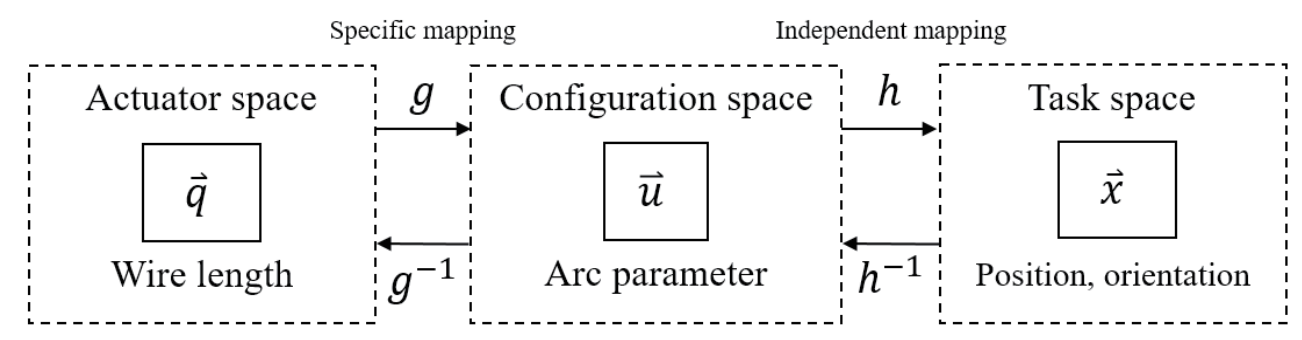

Figure 10. The commonly used kinematic mapping of continuum section. 
the continuum section. In the configuration space, curvature $(\kappa)$ and bending direction $(\varphi)$ are usually used to describe the shape of the continuum section, as shown in Figure 11.

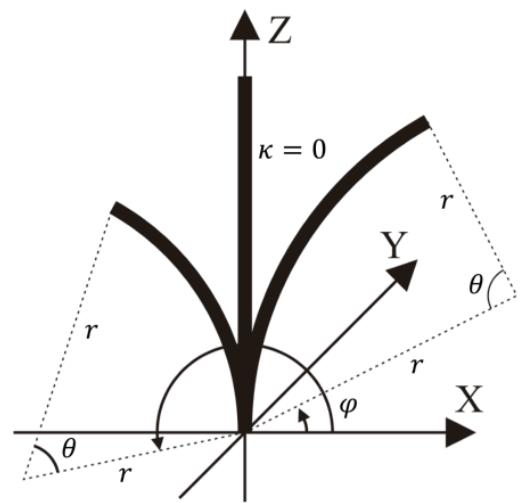

Figure 11. Illustration of the configuration space domain using curvature and bending direction as variables. When $\kappa=0$, the continuum section aligns with the z-axis.

The specific mapping $g$ and independent mapping $h$ can be written as these equations, according to this study (Jones and Walker, 2006).

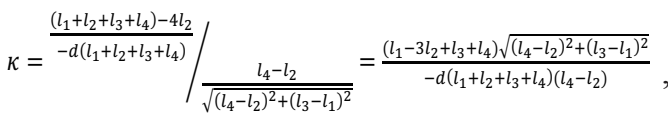

$$
\begin{aligned}
& \varphi=\tan ^{-1}\left(\frac{l_{3}-l_{1}}{l_{4}-l_{2}}\right), \\
& T=\left(\begin{array}{cccc}
\cos ^{2} \varphi(\cos \kappa s-1)+1 & \sin \varphi \cos \varphi(\cos \kappa s-1) & \cos \varphi \sin \kappa s & \frac{\cos \varphi(1-\cos \kappa s)}{k} \\
\sin \varphi \cos \varphi(\cos \kappa s-1) & \cos ^{2} \varphi(1-\cos \kappa s)+\cos \kappa s & \sin \varphi \sin \kappa s & \frac{\sin \varphi(1-\cos \kappa s)}{k} \\
-\cos \varphi \sin \kappa s & -\sin \varphi \sin \kappa s & \cos \kappa s & \frac{\sin \kappa s}{\kappa} \\
0 & 0 & 0 & 1
\end{array}\right),
\end{aligned}
$$

where $l_{1}$ to $l_{4}$ is the length of each wire and $T$ is the transformation matrix for the tip position and orientation of the continuum section. Analogous to conventional kinematic analysis, the velocity kinematic is given by

$$
\dot{\vec{x}}=J \dot{\vec{q}},
$$

where $\dot{\vec{x}}$ is the derivative of the task space vector, $\dot{\vec{q}}$ is the derivative of actuator space vector, $J$ is the Jacobian matrix. This robotic system involves two kinematic mappings, $g$ and $h$ given in the previous sub-section. Therefore, the velocity kinematic of the whole system becomes $\dot{\vec{x}}=J(\vec{u}) J(\vec{q}) \dot{\vec{q}}$, where $J(\vec{q})$ is the Jacobian matrix of the manipulator specific mapping and $J(\vec{u})$ is the Jacobian matrix of the manipulator independent mapping. $J(\vec{q})$ is given by the following:

$$
J(\vec{q})=\frac{\partial \vec{u}}{\partial \vec{q}} .
$$

The Jacobian for the position is then given by

$$
\begin{aligned}
& J_{p}(\vec{u})=\left(\begin{array}{lll}
\frac{\partial x}{\partial \varphi} & \frac{\partial x}{\partial \varphi} & \frac{\partial x}{\partial \varphi} \\
\frac{\partial y}{\partial \kappa} & \frac{\partial y}{\partial \kappa} & \frac{\partial y}{\partial \kappa} \\
\frac{\partial z}{\partial s} & \frac{\partial z}{\partial s} & \frac{\partial z}{\partial s}
\end{array}\right) \\
& =\left(\begin{array}{ccc}
\frac{\sin \varphi \cos (\kappa s)-1}{\kappa} & \frac{\left(2 \sin ^{2}\left(\frac{\varphi}{2}\right)-1\right)\left(2 \sin ^{2}\left(\frac{\kappa s}{2}\right)-\kappa s(\sin (\kappa s))\right)}{\kappa^{2}} & \sin (\kappa s) \cos \varphi \\
-\frac{\cos \varphi \cos (\kappa s)-1}{\kappa} & -\frac{\sin \varphi\left(2 \sin ^{2}\left(\frac{\kappa s}{2}\right)-\kappa s(\sin (\kappa s))\right)}{\kappa^{2}} & \sin (\kappa s) \sin \varphi \\
0 & -\frac{\sin ^{2}(\kappa s)-\kappa s(\cos (\kappa))}{\kappa^{2}} & \cos (\kappa s)
\end{array}\right) .
\end{aligned}
$$

It is obvious that if $\kappa=0$, some terms are undefined, which turns out the singularity of the Jacobian. There are some solutions to this singularity problem (Chiaverini et al., 1994; Jones and Walker, 2007), but their methods reduce model accuracy and increase computational complexity. An axis-angle rotational geometry is introduced to replace this configuration space representation.

As shown in Figure 12, the shape of the continuum section is represented by $\vec{\theta}=\left[\theta_{x} \theta_{y}\right]^{T}$, where $\theta_{x}$ and $\theta_{y}$ are the projection angle of the continuum section on the $\mathrm{x}$ - and $\mathrm{y}$-axis respectively.

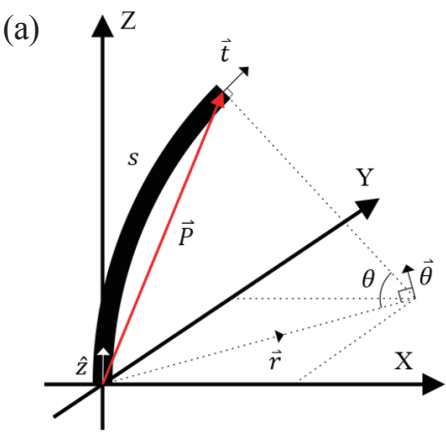

(b)

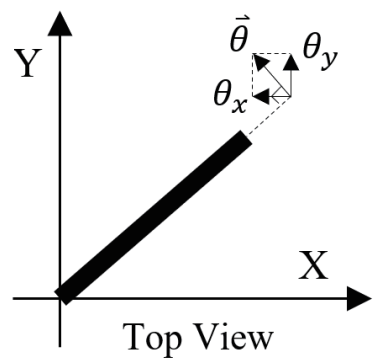

Figure 12. (a) Illustration of the coordinate system of the continuum section using axis-angle rotational geometry. (b) The top view of the coordinate system for the illustration of $\vec{\theta}$ and its components $\theta_{x}$ and $\theta_{y}$. 
In short, the overall Jacobian from actuator space to task space is:

$$
\begin{aligned}
& \bar{J}=\bar{J}_{t}(\vec{\theta}) \bar{J}(\vec{q}) \\
& =\left(\begin{array}{c}
\frac{\sin \left(\frac{\sqrt{\left(l_{1}-s\right)^{2}+\left(l_{2}-s\right)^{2}}}{d}\right)\left(l_{1}-s\right)\left(l_{2}-s\right)}{\left(\sqrt{\left(l_{1}-s\right)^{2}+\left(l_{2}-s\right)^{2}}\right)^{3}}-\frac{\cos \left(\frac{\sqrt{\left(l_{1}-s\right)^{2}+\left(l_{2}-s\right)^{2}}}{d}\right)\left(l_{1}-s\right)\left(l_{2}-s\right)}{d\left(\left(l_{1}-s\right)^{2}+\left(l_{2}-s\right)^{2}\right)} \\
\frac{\sin \left(\frac{\sqrt{\left(l_{1}-s\right)^{2}+\left(l_{2}-s\right)^{2}}}{d}\right)}{\sqrt{\left(l_{1}-s\right)^{2}+\left(l_{2}-s\right)^{2}}}-\frac{\sin \left(\frac{\sqrt{\left(l_{1}-s\right)^{2}+\left(l_{2}-s\right)^{2}}}{d}\right)\left(l_{1}-s\right)\left(l_{2}-s\right)}{\left(\sqrt{\left(l_{1}-s\right)^{2}+\left(l_{2}-s\right)^{2}}\right)^{3}}+\frac{\cos \left(\frac{\sqrt{\left(l_{1}-s\right)^{2}+\left(l_{2}-s\right)^{2}}}{d}\right)\left(l_{1}-s\right)\left(l_{2}-s\right)}{d\left(\left(l_{1}-s\right)^{2}+\left(l_{2}-s\right)^{2}\right)} \\
-\frac{\sin \left(\frac{\sqrt{\left(l_{1}-s\right)^{2}+\left(l_{2}-s\right)^{2}}}{d}\right)\left(l_{1}-s\right)}{d \sqrt{\left(l_{1}-s\right)^{2}+\left(l_{2}-s\right)^{2}}}
\end{array}\right. \\
& \left.\frac{\sin \left(\frac{\sqrt{\left(l_{1}-s\right)^{2}+\left(l_{2}-s\right)^{2}}}{d}\right)\left(l_{1}-s\right)\left(l_{2}-s\right)}{\left(\sqrt{\left(l_{1}-s\right)^{2}+\left(l_{2}-s\right)^{2}}\right)^{3}}-\frac{\cos \left(\frac{\sqrt{\left(l_{1}-s\right)^{2}+\left(l_{2}-s\right)^{2}}}{d}\right)\left(l_{1}-s\right)\left(l_{2}-s\right)}{d\left(\left(l_{1}-s\right)^{2}+\left(l_{2}-s\right)^{2}\right)}-\frac{\sin \left(\frac{\sqrt{\left(l_{1}-s\right)^{2}+\left(l_{2}-s\right)^{2}}}{d}\right)}{\sqrt{\left(l_{1}-s\right)^{2}+\left(l_{2}-s\right)^{2}}}\right) \\
& \frac{\cos \left(\frac{\sqrt{\left(l_{1}-s\right)^{2}+\left(l_{2}-s\right)^{2}}}{d}\right)\left(l_{1}-s\right)\left(l_{2}-s\right)}{d\left(\left(l_{1}-s\right)^{2}+\left(l_{2}-s\right)^{2}\right)}-\frac{\sin \left(\frac{\sqrt{\left(l_{1}-s\right)^{2}+\left(l_{2}-s\right)^{2}}}{d}\right)\left(l_{1}-s\right)\left(l_{2}-s\right)}{\left(\sqrt{\left(l_{1}-s\right)^{2}+\left(l_{2}-s\right)^{2}}\right)^{3}} \\
& -\frac{\sin \left(\frac{\sqrt{\left(l_{1}-s\right)^{2}+\left(l_{2}-s\right)^{2}}}{d}\right)\left(l_{2}-s\right)}{d \sqrt{\left(l_{1}-s\right)^{2}+\left(l_{2}-s\right)^{2}}}
\end{aligned}
$$

When taking the limit at zero curvature situation, we find that the Jacobian is well-defined, in which the continuum section does not have a singularity.

$$
\lim _{l_{2 \rightarrow s}} \lim _{l_{1 \rightarrow s}} \bar{J}=\left(\begin{array}{cc}
0 & \frac{1}{d} \\
-\frac{1}{d} & 0 \\
0 & 0
\end{array}\right) .
$$

The detailed equation will be provided in the appendix for reference.

\section{Experimentations}

\subsection{Feedback controller}

The block diagram for the feedback controller using new configuration space and the corresponding experimental setup is shown in Figures 13 and 14, respectively. A three-point IR reflective marker was attached at the distal end of the continuum manipulator to track the tip position and orientation of the continuum manipulator. The sheath holder and the driving unit were fixed such that the tendon of the manipulator can be directly driven by the motor. Both the optical tracker data collection interface and controller were implemented in a PC using C/ $\mathrm{C}++$ in a real-time setting. The continuum manipulator was commanded to follow a circular trajectory both without and with the proposed feedback controller.

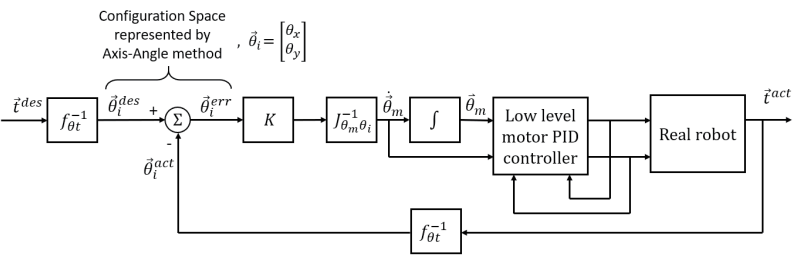

Figure 13. Block diagram of the feedback controller.

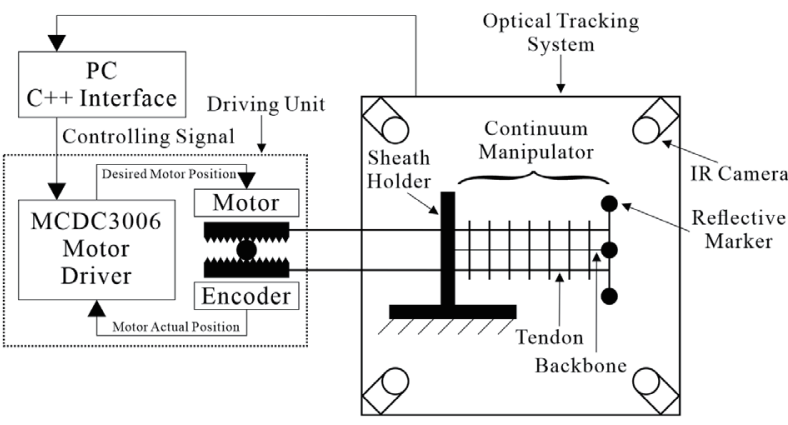

Figure 14. Experimental setup of the feedback controller.

Figure 15 and Table 1 show the typical result and the statics of the experiment. The feedback controller reduces the mean absolute error and the maximum error by $77 \%$ and $68 \%$, respectively, from open-loop control. This proves the correctness and robustness of this feedback controller. 
(a)

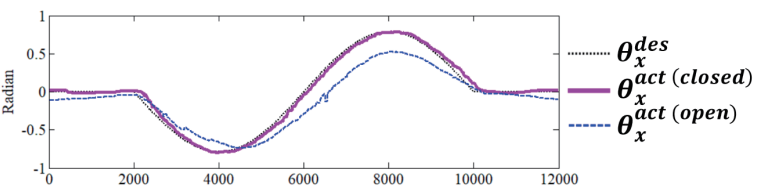

(b)

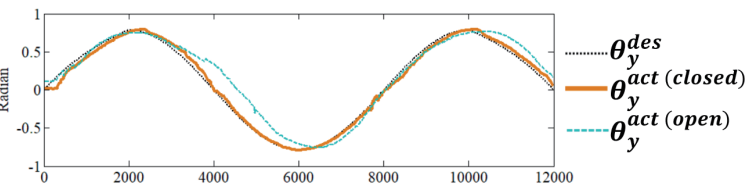

(c)

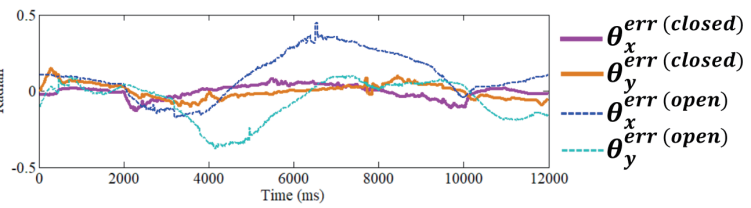

Figure 15. The typical experimental result with and without feedback controller.

Table 1. The experimental statics of the feedback controller.

\begin{tabular}{lccc}
\hline & $\begin{array}{c}\text { Mean } \\
\text { absolute error }\end{array}$ & $\begin{array}{c}\text { Maximum } \\
\text { error }\end{array}$ & SD \\
\hline Open loop & $0.0785 \mathrm{rad}$ & $0.482 \mathrm{rad}$ & $0.0204 \mathrm{rad}$ \\
\hline Closed loop & $0.0181 \mathrm{rad}$ & $0.150 \mathrm{rad}$ & $0.0067 \mathrm{rad}$ \\
\hline
\end{tabular}

\subsection{Clinical experimentation}

Three in-vivo experiments were conducted on the live porcine model. The procedure was similar to the previous ex-vivo experiments (Lau et al., 2016). An around 20-40 $\mathrm{mm}$ diameter area in the stomach was marked and the time to complete the dissection of this area was recorded. Figure 16 shows the experimental setup of this in-vivo trial. The robotic arms were inserted into the pig stomach by passing through the endoscopic platform. The surgeon used the master console to control the robotic arms through the view from the endoscopic camera. A nurse helped him to adjust the camera viewing angle. Figure 17 shows the typical moment when the lifter was lifting up the dissecting tissue layer.

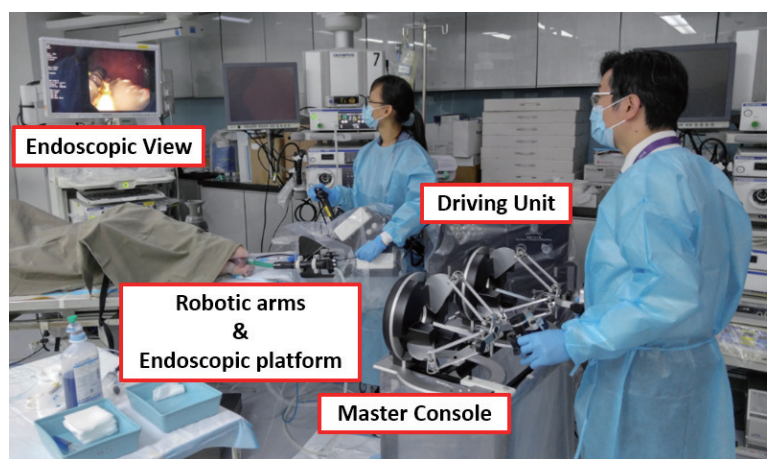

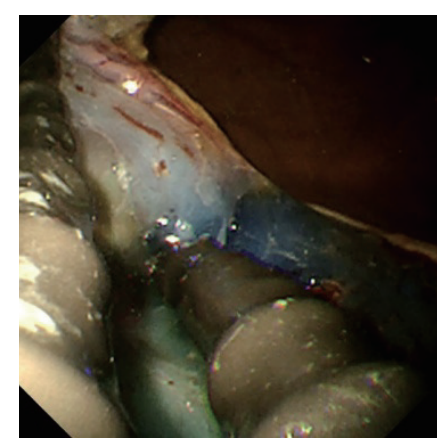

Figure 17. The typical moment when the lifter was lifting up the tissue.

The average time of these three experiments was about $28 \pm 10$ minutes. For conventional ESD, the reported time is $84 \pm 54.6$ minutes. In all trials, no bleeding or perforation happened.

\section{Conclusion}

An endoscopic surgery robotic platform was built for Endoscopic Submucosal Dissection. It has been proved in in-vivo experiments that it increases the safety and efficiency of ESD over the conventional method. An advanced robotic platform was built based on the prototype platform, in which the flexibility and commercialisation potential are improved.

\section{Appendix}

In this section, a new coordinate system for configuration space is presented. The position and the pointing direction of the continuum manipulator tip is found by the axis-angle rotational method, a well-known technique for finding the rotational information between two vectors.

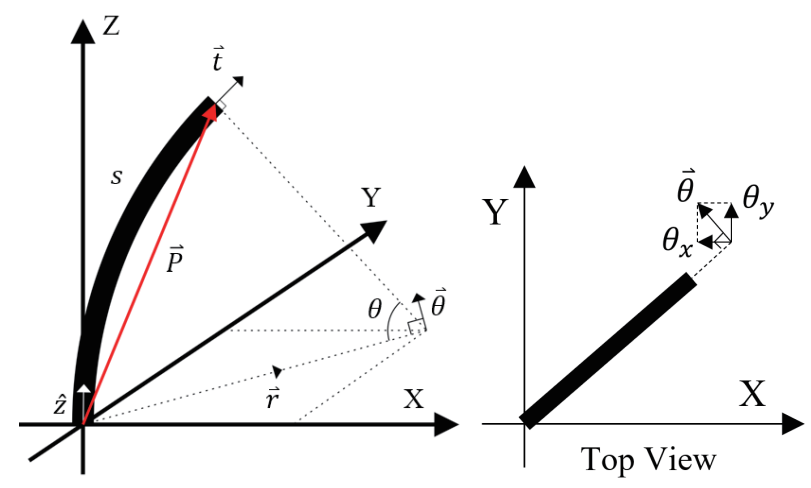

Figure A1. The geometry representation of the continuum manipulator in the axis-angle rotational representation.

Figure 16. The experimental setup of the in-vivo trial. 
In the above illustration, the arc is no longer represented by the arc parameters $\varphi, \kappa$. It is represented by a vector $\vec{\theta}$ laying on the XY-plane which passes through the centre of the arc. $\vec{\theta}$ contains two components, $\theta_{x}$ and $\theta_{y}$, i.e., $\vec{\theta}=\left[\theta_{x} \theta_{y}\right]^{T}$, as illustrated in the top view picture on the right-hand side. The magnitude of $\vec{\theta}$ defines the bending angle of the arc, i.e.

$$
\theta=\|\vec{\theta}\|=\sqrt{\theta_{x}^{2}+\theta_{y}^{2}} .
$$

The unit vector of $\vec{\theta}$ is given by

$$
\hat{\theta}=\frac{\vec{\theta}}{\|\vec{\theta}\|}=\frac{\vec{\theta}}{\sqrt{\theta_{x}^{2}+\theta_{y}^{2}}} .
$$

The pointing direction $\vec{t}$ and position vector $\vec{P}$ are calculated by using axis-angle rotational method between the unit $Z$ vector $\hat{z}$ and $\hat{\theta}$. In other word, it can be imagined that $\hat{z}$ is rotating about $\vec{\theta}$ by $\theta$ to become $\vec{t}$.

Thus, the kinematic mapping of the continuum manipulator becomes the following.

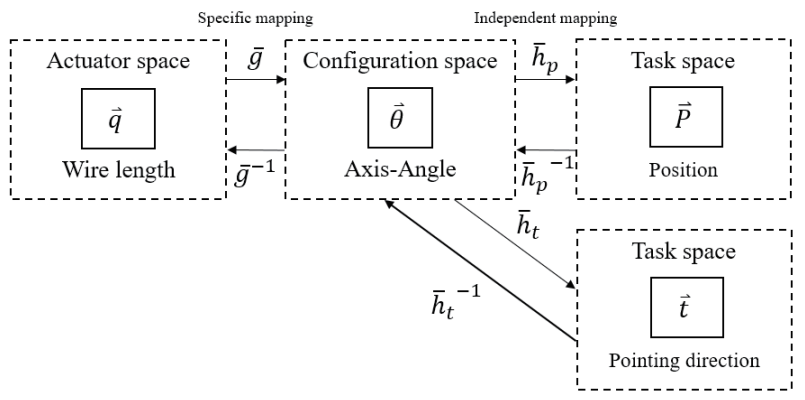

Figure A2. The proposed kinematic mapping of the continuum manipulator.

$\bar{h}_{p}$ defines the independent mapping from configuration space to the position in the task space; and $\bar{h}_{t}$ defines the independent mapping from configuration space to the pointing direction in the task space.

To find the independent mapping, firstly let $K$ be the cross-product matrix of $\hat{\theta}$, then,

$$
K=\frac{\left(\begin{array}{ccc}
0 & 0 & \theta_{y} \\
0 & 0 & -\theta_{x} \\
-\theta_{y} & \theta_{x} & 0
\end{array}\right)}{\sqrt{\theta_{x}^{2}+\theta_{y}^{2}}} .
$$

By the definition of axis-angle rotational method, the rotation transformation matrix $R$ of $\hat{z}$ about $\vec{\theta}$ is

$$
\begin{aligned}
& R=I+\sin \theta \cdot K+(1-\cos \theta) \cdot K^{2} \\
& =\left(\begin{array}{lll}
1 & 0 & 0 \\
0 & 1 & 0 \\
0 & 0 & 1
\end{array}\right)+\frac{\sin \theta}{\sqrt{\theta_{x}{ }^{2}+\theta_{y}}}\left(\begin{array}{ccc}
0 & 0 & \theta_{y} \\
0 & 0 & -\theta_{x} \\
-\theta_{y} & \theta_{x} & 0
\end{array}\right)+\frac{(1-\cos \theta)}{\left(\sqrt{\theta_{x}{ }^{2}+\theta_{y}{ }^{2}}\right)^{2}}\left(\begin{array}{ccc}
0 & 0 & \theta_{y} \\
0 & 0 & -\theta_{x} \\
-\theta_{y} & \theta_{x} & 0
\end{array}\right)^{2} \\
& =\left(\begin{array}{ccc}
\frac{\theta_{y}{ }^{2}(\cos \theta-1)}{\theta^{2}}+1 & -\frac{\theta_{x} \theta_{y}(\cos \theta-1)}{\theta^{2}} & \frac{\theta_{y} \sin \theta}{\theta} \\
-\frac{\theta_{x} \theta_{y}(\cos \theta-1)}{\theta^{2}} & \frac{\theta_{x}{ }^{2}(\cos \theta-1)}{\theta^{2}}+1 & -\frac{\theta_{x} \sin \theta}{\theta} \\
-\frac{\theta_{y} \sin \theta}{\theta} & \frac{\theta_{x} \sin \theta}{\theta} & (\cos \theta-1)\left(\frac{\theta_{x}{ }^{2}}{\theta^{2}}+\frac{\theta_{y}{ }^{2}}{\theta^{2}}\right)+1
\end{array}\right),
\end{aligned}
$$

where $I$ is the identity matrix and $\theta=\sqrt{\theta_{x}{ }^{2}+\theta_{y}{ }^{2}}$.

$\vec{r}$ is the radius of the arc. It is calculated by the crossproduct of $\hat{\theta}$ and $\hat{z}$, i.e.,

$$
\vec{r}=\|\vec{r}\| \cdot(\hat{\theta} \times \hat{z})=\frac{\|\vec{r}\|}{\sqrt{\theta_{x}^{2}+\theta_{y}^{2}}} \cdot(\vec{\theta} \times \hat{z}),
$$

where $\|\vec{r}\|=\frac{s}{\|\vec{\theta}\|}=\frac{s}{\sqrt{\theta_{x}^{2}+\theta_{y}^{2}}}$.

Therefore,

$$
\begin{aligned}
\vec{r} & =\frac{s}{\left(\sqrt{\theta_{x}^{2}+\theta_{y}^{2}}\right)^{2}}\left(\left(\begin{array}{c}
\theta_{x} \\
\theta_{y} \\
0
\end{array}\right) \times\left(\begin{array}{l}
0 \\
0 \\
1
\end{array}\right)\right) \\
& =\frac{s}{\theta_{x}^{2}+\theta_{y}{ }^{2}}\left(\begin{array}{c}
\theta_{y} \\
-\theta_{x} \\
0
\end{array}\right) \\
& =\left(\begin{array}{c}
\frac{s \theta_{y}}{\theta_{x}^{2}+\theta_{y}^{2}} \\
\frac{-s \theta_{x}}{\theta_{x}^{2}+\theta_{y}{ }^{2}} \\
0
\end{array}\right) .
\end{aligned}
$$

The position of the tip is found by the following transformation sequence: (1) translating the centre of the arc to origin; (2) rotating about origin by $\theta$; (3) translating back to the centre of the arc.

$$
\begin{aligned}
& \vec{P}=R \cdot(-\vec{r})+\vec{r} \\
& =R \cdot\left(-\left(\begin{array}{c}
\frac{s \theta_{y}}{\theta_{x}^{2}+\theta_{y}{ }^{2}} \\
\frac{-s \theta_{x}}{\theta_{x}^{2}+\theta_{y}{ }^{2}} \\
0
\end{array}\right)\right)+\left(\begin{array}{c}
\frac{s \theta_{y}}{\theta_{x}^{2}+\theta_{y}{ }^{2}} \\
\frac{-s x_{x}}{\theta_{x}^{2}+\theta_{y}{ }^{2}} \\
0
\end{array}\right) \\
& =s \cdot\left(\begin{array}{c}
\frac{1-\cos \sqrt{\theta_{x}{ }^{2}+\theta_{y}{ }^{2}}}{\theta_{x}{ }^{2}+\theta_{y}{ }^{2}} \theta_{y} \\
\frac{\cos \sqrt{\theta_{x}^{2}+\theta_{y}{ }^{2}-1}}{\theta_{x}{ }^{2}+\theta_{y}{ }^{2}} \theta_{x} \\
\frac{\sin \sqrt{\theta_{x}{ }^{2}+\theta_{y}{ }^{2}}}{\sqrt{\theta_{x}{ }^{2}+\theta_{y}{ }^{2}}}
\end{array}\right) .
\end{aligned}
$$


The pointing direction $\vec{t}$ is calculated by multiplying $R$ by $\hat{z}$.

$$
\begin{aligned}
\vec{t} & =R \cdot \hat{z} \\
& =R \cdot\left(\begin{array}{l}
0 \\
0 \\
1
\end{array}\right) \\
& =\left(\begin{array}{l}
\frac{\sin \sqrt{\theta_{x}^{2}+\theta_{y}^{2}}}{\sqrt{\theta_{x}^{2}+\theta_{y}^{2}}} \theta_{y} \\
-\frac{\sin \sqrt{\theta_{x}^{2}+\theta_{y}^{2}}}{\sqrt{\theta_{x}^{2}+\theta_{y}^{2}}} \theta_{x} \\
\cos \sqrt{\theta_{x}^{2}+\theta_{y}^{2}}
\end{array}\right) .
\end{aligned}
$$

Here, we successfully define $\bar{h}_{t}$ and $\bar{h}_{p}$.

To find $\bar{h}_{t}{ }^{-1}$, we can compute the total bending angle as

$$
\theta=\operatorname{atan} 2\left(\sqrt{t_{x}^{2}+t_{y}^{2}}, t_{z}\right)
$$

where $t_{x}, t_{y}, t_{z}$ are the $\mathrm{x}, \mathrm{y}, \mathrm{z}$ component of the pointing direction vector $\vec{t}$. Notice that $\sqrt{\theta_{x}{ }^{2}+\theta_{y}{ }^{2}}$. By rearranging the terms in $\bar{h}_{t}$, we have,

$$
\bar{h}_{t}^{-1}\left\{\begin{array}{l}
\theta_{x}=-t_{y} \frac{\theta}{\sin \theta} \\
\theta_{y}=t_{x} \frac{\theta}{\sin \theta} .
\end{array}\right.
$$

To find $\bar{h}_{p}{ }^{-1}$, we again need to compute the total bending first.

$$
\begin{aligned}
& \sqrt{p_{x}^{2}+p_{y}^{2}}=s \frac{1-\cos \theta}{\theta}=\frac{s}{\theta}\left(1-1+2 \sin ^{2} \frac{\theta}{2}\right)=\frac{2 s}{\theta} \sin ^{2} \frac{\theta}{2} \\
& p_{z}=s \frac{\sin \theta}{\theta}=\frac{2 s}{\theta} \sin \frac{\theta}{2} \cos \frac{\theta}{2} \\
& \Rightarrow \frac{\sqrt{p_{x}^{2}+p_{y}^{2}}}{p_{z}}=\frac{\sin \frac{\theta}{2}}{\cos \frac{\theta}{2}}=\tan \frac{\theta}{2} .
\end{aligned}
$$

Therefore,

$$
\theta=2 \operatorname{atan} 2\left(\sqrt{p_{x}^{2}+p_{y}^{2}}, p_{z}\right)
$$

Thus,

$$
\bar{h}_{p}^{-1}\left\{\begin{array}{c}
\theta_{x}=-p_{y} \frac{\theta}{\sqrt{p_{x}^{2}+p_{y}^{2}}} \\
\theta_{y}=p_{x} \frac{\theta}{\sqrt{p_{x}^{2}+p_{y}^{2}}} .
\end{array}\right.
$$

To find the manipulator dependent mapping $\bar{g}$ and $\bar{g}^{-1}$, consider the geometric analysis of the manipulator in below figure.

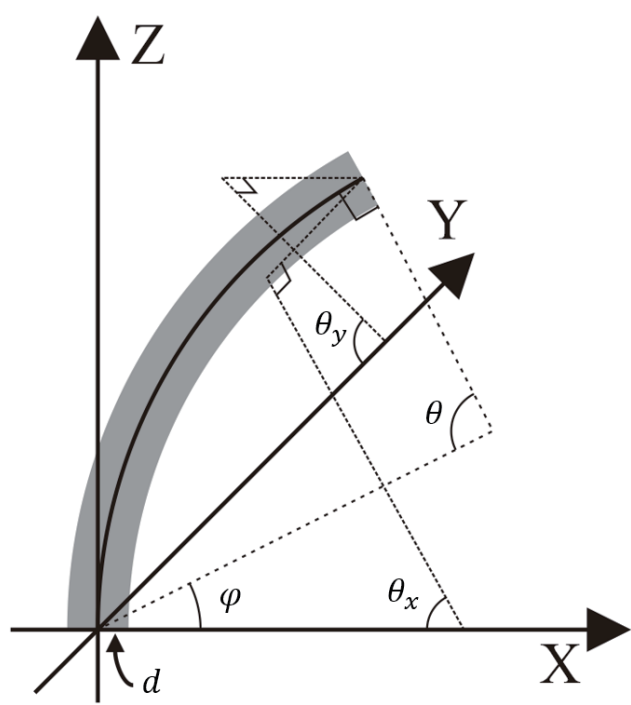

Figure A3. The geometric representation of $\theta_{x}$ and $\theta_{y}$.

According to the derivation in the last sub-section, we have the following relations.

$$
\begin{aligned}
& \left(\begin{array}{l}
x \\
y
\end{array}\right)=\left(\begin{array}{l}
\frac{1-\cos \theta}{\theta^{2}} \theta_{y} \\
\frac{\cos \theta-1}{\theta^{2}} \theta_{x}
\end{array}\right) \\
& \theta^{2}=\theta_{x}{ }^{2}+\theta_{y}{ }^{2}
\end{aligned}
$$

where $x$ and $y$ are the $\mathrm{x}$ and $\mathrm{y}$ components of the distal tip of the continuum manipulator respectively.

From the above figure, we have

$$
\begin{aligned}
& \tan \varphi=\frac{y}{x}=\frac{\frac{\cos \theta-1}{\theta^{2} \theta_{x}}}{\frac{1-\cos \theta}{\theta^{2}} \theta_{y}}=-\frac{\theta_{x}}{\theta_{y}} \\
& s=r \theta \\
& l_{i}=r_{i} \theta \\
& \Rightarrow s-l_{i}=\theta\left(r-r_{i}\right)
\end{aligned}
$$

where $s$ is the length of the backbone, $r$ is the radius of the arc of the backbone, $l_{i}$ is the length of the $i$ th actuator wire, $r_{i}$ is the radius of the arc of $i$ th actuator wire. Substituting $r_{i}=r-d \cos \varphi_{i}$

$$
s-l_{i}=\theta d \cos \varphi_{i} .
$$


For $i=1,2$

$$
\left\{\begin{array}{c}
s-l_{1}=\theta d \cos \varphi_{1}=\theta d \cos \left(90^{\circ}-\varphi\right)=\theta d \sin \varphi \\
s-l_{2}=\theta d \cos \varphi_{2}=\theta d \cos \left(180^{\circ}-\varphi\right)=-\theta d \cos \varphi
\end{array}\right.
$$

$$
\Rightarrow\left\{\begin{array}{l}
s-l_{1}=d \sqrt{\theta_{x}^{2}+\theta_{y}^{2}} \frac{-\theta_{x}}{\sqrt{\theta_{x}{ }^{2}+\theta_{y}^{2}}}=-d \theta_{x} \\
s-l_{2}=-d \sqrt{\theta_{x}^{2}+\theta_{y}^{2}} \frac{\theta_{y}}{\sqrt{\theta_{x}^{2}+\theta_{y}^{2}}}=-d \theta_{y}
\end{array}\right.
$$

In summary,

$$
\begin{gathered}
\bar{g}=\left\{\begin{array}{l}
\theta_{x}=\frac{l_{1}-s}{d} \\
\theta_{y}=\frac{l_{2}-s}{d}
\end{array}\right. \\
\bar{g}^{-1}=\left\{\begin{array}{l}
l_{1}=d \theta_{x} \\
l_{2}=d \theta_{y}
\end{array}\right.
\end{gathered}
$$

The Jacobian of this kinematic mapping is written by

$$
\dot{\vec{x}}=\bar{J}_{t}(\vec{\theta}) \bar{J}(\vec{q}) \dot{\vec{q}}
$$

where $\bar{J}(\vec{q})$ is the Jacobian from actuator space to configuration space and $\bar{J}_{t}(\vec{\theta})$ is the Jacobian from configuration space to pointing direction in task space.

$$
\begin{aligned}
& \bar{J}_{t}(\vec{\theta})=\frac{\partial \vec{t}}{\partial \vec{\theta}}=\left(\begin{array}{ll}
\frac{\partial t_{x}}{\partial \theta_{x}} & \frac{\partial t_{x}}{\partial \theta_{y}} \\
\frac{\partial t_{y}}{\partial \theta_{x}} & \frac{\partial t_{y}}{\partial \theta_{y}} \\
\frac{\partial t_{z}}{\partial \theta_{x}} & \frac{\partial t_{z}}{\partial \theta_{y}}
\end{array}\right) \\
& =\left(\begin{array}{cc}
\frac{\theta_{x} \theta_{y} \cos \theta}{\theta^{2}}-\frac{\theta_{x} \theta_{y} \sin \theta}{\theta^{3}} & \frac{\theta_{y}{ }^{2} \cos \theta}{\theta^{2}}+\frac{\theta_{x}{ }^{2} \sin \theta}{\theta^{3}} \\
-\frac{\theta_{x}{ }^{2} \cos \theta}{\theta^{2}}-\frac{\theta_{y}{ }^{2} \sin \theta}{\theta^{3}} & \frac{\theta_{x} \theta_{y} \sin \theta}{\theta^{3}}-\frac{\theta_{x} \theta_{y} \cos \theta}{\theta^{2}} \\
-\frac{\theta_{x} \sin \theta}{\theta} & -\frac{\theta_{y} \sin \theta}{\theta}
\end{array}\right) \\
& \bar{J}(\vec{q})=\frac{\partial \vec{\theta}}{\partial \vec{q}}=\left(\begin{array}{cc}
\frac{\partial \theta_{x}}{\partial l_{1}} & \frac{\partial \theta_{x}}{\partial l_{2}} \\
\frac{\partial \theta_{y}}{\partial l_{1}} & \frac{\partial \theta_{y}}{\partial l_{2}}
\end{array}\right)=\left(\begin{array}{cc}
\frac{1}{d} & 0 \\
0 & \frac{1}{d}
\end{array}\right)
\end{aligned}
$$

The overall Jacobian:

$$
\bar{J}=\bar{J}_{t}(\vec{\theta}) \bar{J}(\vec{q})
$$

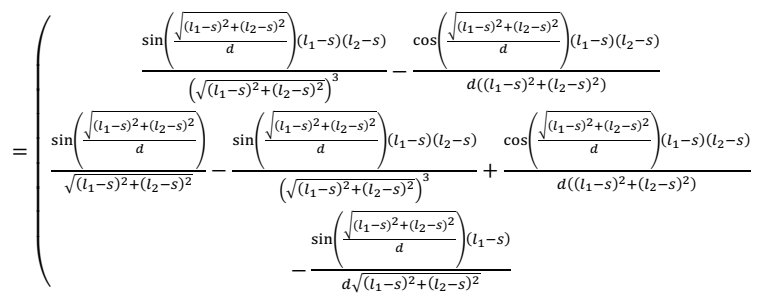

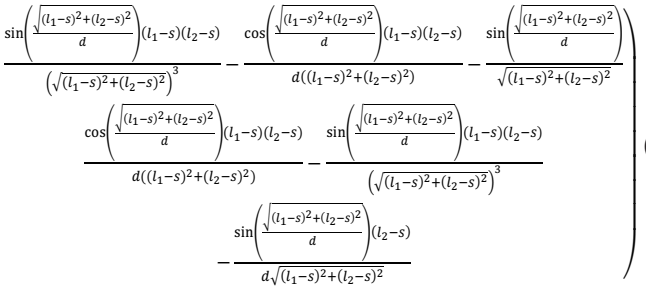

The singularity happened in the typical coordinate when the curvature is. In the axis-angle rotational method, we can take the limit at $l_{1}=s$ and $l_{2}=s$ to test it.

$$
\lim _{l_{2 \rightarrow s}} \lim _{1 \rightarrow s} \bar{J}=\left(\begin{array}{cc}
0 & \frac{1}{d} \\
-\frac{1}{d} & 0 \\
0 & 0
\end{array}\right) .
$$

It shows that the Jacobian is well-defined when the continuum manipulator is in straight posture; and it is obvious that it is also well-defined in the domain.

The stability of the kinematic feedback controller is proofed using the Lyapunov function. First, let's define a configuration space error $\vec{\theta}_{r}$ as the deviation of the real configuration space vector $\vec{\theta}_{e}$ and the desired configuration space vector $\vec{\theta}_{d}$. Thus,

$$
\vec{\theta}_{e}=\vec{\theta}_{d}-\vec{\theta}_{r}
$$

The time derivative of the above equation gives,

$$
\dot{\vec{\theta}}_{e}=\dot{\vec{\theta}}_{d}-\dot{\vec{\theta}}_{r}=\dot{\vec{\theta}}_{d}-\eta \bar{J}(\vec{q}) \dot{\vec{q}}_{c o m m}
$$

where $\eta$ is the modeling uncertainties with a positive scalar, $\bar{J}(q)$ is the Jacobian matrix from actuator space to configuration space using axis-angle method, and $\dot{\vec{q}}_{\text {comm }}$ is the commanded actuator velocity.

From the above control loop, we have

$\dot{\vec{q}}_{c o m m}=\bar{J}(\vec{q})^{-1} K \vec{\theta}_{e}$.

Combining the above two equations, we get

$\dot{\vec{\theta}}_{e}=\dot{\vec{\theta}}_{d}-\eta K \vec{\theta}_{e}$

The Lyapunov function is defined as

$V\left(\vec{\theta}_{e}\right)=\frac{1}{2} \vec{\theta}_{e}^{T} K \vec{\theta}_{e}$

And its derivative is

$$
\begin{aligned}
& \dot{V}\left(\vec{\theta}_{e}\right)=\vec{\theta}_{e}{ }^{T} K \dot{\vec{\theta}}_{e}=\vec{\theta}_{e}{ }^{T} K\left(\dot{\vec{\theta}}_{d}-\eta K \vec{\theta}_{e}\right) \\
& =\vec{\theta}_{e}{ }^{T} K \dot{\vec{\theta}}_{d}-\eta \vec{\theta}_{e}{ }^{T} K K \vec{\theta}_{e}
\end{aligned}
$$


In the case of trajectory following regulation, $\dot{\vec{\theta}}_{d}=0$ ; therefore $\vec{\theta}_{e}{ }^{T} K \dot{\vec{\theta}}_{d}=0$. Since $\eta$ and $K$ are positive and $\vec{\theta}_{e}^{T} \vec{\theta}_{e}$ gives out positive definite result, thus

$$
\eta \vec{\theta}_{e}^{T} K K \vec{\theta}_{e}>0
$$

As the result, the stability of the controller is proofed by the following finding:

$$
\dot{V}\left(\vec{\theta}_{e}\right)=-\eta \vec{\theta}_{e}^{T} K K \vec{\theta}_{e}<0
$$

\section{Acknowledgement}

The work was supported by the ITSP Tier 2 Platform under Grant ITS/252/13FP and ITS/178/18FP; and the InnoHK funding.

\section{Note on contributors}

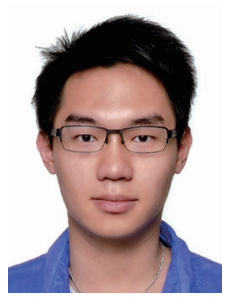

Dr Ka Chun Lau received a B. Eng. and $\mathrm{Ph}$. D. degree in mechanical and automation engineering from The Chinese University of Hong Kong in 2013 and 2017 respectively. He is currently a postdoctoral fellow in mechanical and automation engineering and the mechanical engineer in Multiscale Medical Robotics Center at The Chinese University of Hong Kong.

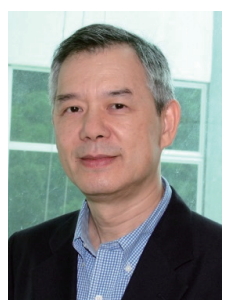

Prof Yeung Yam is a Professor at the Department of Mechanical and Automation Engineering, The Chinese University of Hong Kong (CUHK). He received his bachelor degree in Physics from CUHK, and his M.Sc. and Sc.D. degrees in Aeronautics and Astronautics from the Massachusetts Institute of Technology. He is the current Director of the Intelligent Control Systems Laboratory (LCSL), and also the CUHKBeijing Institute of Technology Joint Research Center for Optomechatronics Design and Engineering (JRCODE). He is the Associate Master of the Lee Woo Sing College, one of the nine colleges in CUHK, and also the Director of the Energy and Environmental Engineering Programme of the University. His general research interests include dynamics modelling and control, dexterous robotics, human skill acquisition and automation systems. He has published over 150 technical papers in his areas of interest.

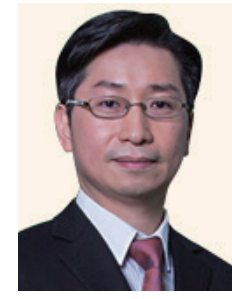

Prof Philip Wai Yan Chiu received a MBChB in 1994 from The Chinese University of Hong Kong (CUHK). He received a FRCS (Edin), FRCS (Edin) (Gen), FHKAM (Surgery) and MD (CUHK) in 1998, 2001, 2001 and 2009 respectively. From 2005-2011, he was an Associate Professor in the Department of Surgery of CUHK. Currently, he is a Professor in the Department of Surgery of CUHK, Director of CUHK Jockey Club Minimally Invasive Surgical Skills Center, Assistant Dean (External Affairs) of Faculty of Medicine of CUHK and Director of CUHK Chow Yuk Ho Technology Center for Innovative Medicine. He was the first surgeon to perform Endoscopic Submucosal Dissection (ESD) for treatment of early GI cancers and Per Oral Endoscopic Myotomy (POEM) for treatment of Achalasia in Hong Kong. His research interests include robotic surgery. He has pioneered performance of robotic esophagectomy for treatment of squamous esophageal cancer and performed the world's first robotic ESD. He contributed to the development of the novel laparoscopic liver retractor and ESD device for treatment of early GI cancers. He is also the author of over 170 peer-reviewed articles and five patents.

\section{References}

[1] Ahn J et al (2021). A highly intuitive and ergonomic redundant joint master device for four-degrees of freedom flexible endoscopic surgery robot. The International Journal of Medical Robotics and Computer Assisted Surgery, 17(1), pp. 1-14.

[2] Asano M (2012). Endoscopic submucosal dissection and surgical treatment for gastrointestinal cancer. World Journal of Gastrointestinal Endoscopy, 4(10), p. 438.

[3] Cao L et al (2019). A novel robotic suturing system for flexible endoscopic surgery. 2019 International Conference on Robotics and Automation (ICRA), pp. 1514-1520.

[4] Cenitagoya G F, Bergh C K and Klinger-Roitman J (1998). A prospective study of gastric cancer. Digestive surgery, 15(4), pp. 317-322.

[5] Chiaverini S, Siciliano B and Egeland O (1994). Review of the Damped Least-Squares Inverse Kinematics with Experiments on an Industrial Robot Manipulator. IEEE Transactions on Control Systems Technology, 2(2), pp. 123-134.

[6] De Donno A et al (2013). Introducing STRAS: A new flexible robotic system for minimally invasive surgery. IEEE International Conference on Robotics and Automation, pp. 1213-1220.

[7] Gotoda T, Yamamoto H and Soetikno R M (2006). Endoscopic submucosal dissection of early gastric cancer. Journal of Gastroenterology, pp. 929-942. 
[8] International Agency for Research on Cancer (2020) Global Cancer Observatory: Cancer Today.

[9] Isomoto H et al (2009). Clinicopathological factors associated with clinical outcomes of endoscopic submucosal dissection for colorectal epithelial neoplasms. Endoscopy, 41(8), pp. 679-683.

[10] Jones B A and Walker I D (2006). Kinematics for multisection continuum robots. IEEE Transactions on Robotics, 22(1), pp. 43-55.

[11] Jones B A and Walker I D (2007). Limitingcase analysis of continuum trunk kinematics. IEEE International Conference on Robotics and Automation, pp. 1363-1368.

[12] Lau K et al (2017). Development of an Endoscopic Surgical Robotic System and From Bench to Animal Studies. Gastrointestinal Endoscopy, 85(5), pp. AB90-AB91.

[13] Lau K C et al (2016). A flexible surgical robotic system for removal of early-stage gastrointestinal cancers by endoscopic submucosal dissection. IEEE Transactions on Industrial Informatics, 12(6), pp. 2365-2374.

[14] Morgan D F K T (2017). Early gastric cancer: Epidemiology, clinical manifestations, diagnosis, and staging, Douglas Morgan. UpToDate.

[15] Mylonas G P et al (2014). CYCLOPS: A versatile robotic tool for bimanual single-access and naturalorifice endoscopic surgery. IEEE International Conference on Robotics and Automation, pp. 24362442.

[16] Oka S et al (2006). Advantage of endoscopic submucosal dissection compared with EMR for early gastric cancer. Gastrointestinal Endoscopy, 64(6), pp. 877-883.

[17] Spaun G O, Zheng B and Swanström L L (2009). A multitasking platform for natural orifice translumenal endoscopic surgery (NOTES): a benchtop comparison of a new device for flexible endoscopic surgery and a standard dual-channel endoscope. Surgical endoscopy, 23(12), p. 2720.

[18] Swanstrom L L et al (2005). Development of a new access device for transgastric surgery. Journal of gastrointestinal surgery : official journal of the Society for Surgery of the Alimentary Tract, 9, pp. 1129-1136; discussion 1136-1137.

[19] Teoh A Y B et al (2010). Difficulties and outcomes in starting endoscopic submucosal dissection. Surgical Endoscopy and Other Interventional Techniques, 24(5), pp. 1049-1054.

[20] Yoshida N et al (2010). Outcome of endoscopic submucosal dissection for colorectal tumours in elderly people. International Journal of Colorectal Disease, 25(4), pp. 455-461.

[21] Yoshida N et al (2013). Therapeutic and diagnostic approaches in colonoscopy. Endoscopy of GI Tract.
[22] Zhou P H, Yao L Q and Qin X Y (2009). Endoscopic submucosal dissection for colorectal epithelial neoplasm. Surgical Endoscopy and Other Interventional Techniques, 23(7), pp. 1546-1551. 